\title{
EchoGéo
}

$17 \mid 2011$

Activités extractives

\section{Lectures croisées sur la Mauritanie du centre et des marges}

Nouakchott. Au carrefour de la Mauritanie et du monde Les Peuls et l'État en Mauritanie

Jérôme Lombard

\section{CpenEdition}

Journals

Édition électronique

URL : https://journals.openedition.org/echogeo/12601

DOI : 10.4000/echogeo.12601

ISSN : 1963-1197

Éditeur

Pôle de recherche pour l'organisation et la diffusion de l'information géographique (CNRS UMR 8586)

Référence électronique

Jérôme Lombard, «Lectures croisées sur la Mauritanie du centre et des marges », EchoGéo [En ligne], 17 | 2011, mis en ligne le 27 septembre 2011, consulté le 11 août 2021. URL : http://

journals.openedition.org/echogeo/12601; DOI : https://doi.org/10.4000/echogeo.12601

Ce document a été généré automatiquement le 11 août 2021.

EchoGéo est mis à disposition selon les termes de la licence Creative Commons Attribution - Pas d'Utilisation Commerciale - Pas de Modification 4.0 International (CC BY-NC-ND) 


\section{Lectures croisées sur la Mauritanie du centre et des marges}

Nouakchott. Au carrefour de la Mauritanie et du monde

Les Peuls et l'État en Mauritanie

Jérôme Lombard

\section{Note de lecture}

1 Il n'est pas si fréquent d'avoir l'opportunité de parler d'un pays africain à partir d'entrées et d'approches a priori diamétralement opposées mais qui, au bout du compte, se rejoignent et donnent une image saisissante des évolutions en cours.

$2700 \mathrm{~km}$ de sable et de brousse séparent les objets abordés dans les deux ouvrages portant sur la Mauritanie, c'est ainsi que Riccardo Ciavolella rappelle ce qui distingue les Peuls du Sud-Est mauritanien de Nouakchott la capitale, observée et analysée par Armelle Choplin. Ces deux mondes se regardent, s'ignorent même, puis progressivement, avec espoir ou dans la douleur, ne forment qu'un seul ensemble, parcouru de traumatismes et de déceptions, mais réel et vivant. R. Ciavolella a décidé d'entreprendre une anthropologie des marges de la Mauritanie, considérées «... non pas comme le lieu de l'État inaccompli mais comme une partie intégrante de celui-ci où ses formes et son autorité sont continuellement recomposées » (30). Et pour cela il choisit le prisme des Fulaabe, ce peuple de pasteurs nomades qui sont venus habiter les terres de l'actuelle Mauritanie durant le $20^{\mathrm{e}}$ siècle ${ }^{1}$ et dont «... la trajectoire périphérique apparaît [...] comme un angle de vue privilégié afin de déceler les logiques et les processus qui se mettent en œuvre avec le développement et la construction du système étatique mauritanien" (49). Ce livre plein de retenue est d'une richesse exceptionnelle et donne à voir la Mauritanie à travers les heurs et malheurs d'une population isolée et pourtant totalement mauritanienne. A l'opposé pourrait-on dire, du moins au début, A. Choplin s'inscrit au centre du pays, c'est-à-dire à l'ouest près de l'océan, au cœur du pouvoir politique, administratif, économique et symbolique, et nous montre comment, d'un fort colonial et de quelques tentes, est née une capitale, 
Nouakchott, ville interface entre société et politique, entre élites et "résidus » (27), ville réputée bâtie dans un espace charnière entre pays des Blancs et pays des Noirs, ville au confluent de l'on shore et de l'off shore, des richesses nationales et de celles de l'extérieur, ville enfin de la mondialisation et ville dans la mondialisation. L'ouvrage de cette géographe est là aussi en phase avec la Mauritanie contemporaine, montrant un État autoritaire aux mains d'une élite politico-commerçante puissante, en butte à la contestation de populations en partie délaissées mais actives.

\section{Frontier mauritanienne}

3 La Mauritanie pourrait se résumer en une histoire de frontières. Les Fulaabe ont choisi les marges pour pouvoir circuler, recherchant la liberté de pâturage en même temps que l'autonomie. Repoussant la frontière des possibles, fuyant les sécheresses, les impôts, le contrôle politique, les colons, ils se déplacèrent plus à l'est, franchissant le fleuve Sénégal et se perdant au $20^{\mathrm{e}}$ siècle dans les plaines de l'Assaba et la vallée du Karakoro. Les vieux disaient, dixit R. Ciavolella (93), « [...] que les colons nous évitent ! ", soulignant par là, et l'ouvrage en est empreint, les formes de résistance, d'autonomie ou d'indépendance d'acteurs apparemment dépolitisés. Ne peut-on voir dans cet évitement permanent trace de "l'homme sans l'occident », ce petit ouvrage écrit il y a près de cent ans par un militaire français attentif à la vie des habitants du pays qu'il conquiert et qui ne lui doit rien².

4 Cette quête de frontière, jusqu'au milieu du siècle dernier, ne croise pas tout de suite celle montante des pouvoirs agissant dans l'Ouest sahélo-saharien. Mais l'espace des Maures, parsemé dès l'époque précoloniale et coloniale de regroupements de tentes, de forts, « espace de la jonction et de la circulation permis par l'existence de villes et l'organisation réticulaire de la société » (Choplin: 51), sous-tend l'apparition de la Mauritanie moderne et de sa capitale qui, progressivement à partir de 1957, rayonne sur l'ensemble du territoire national, par l'intermédiaire du réseau routier, jusqu'à faire reculer puis disparaître les espaces insoumis. On assiste alors à la naissance d'une ville, à son expansion, à la création d'un centre administratif, de quelques industries, à la construction d'un port en eau profonde, en définitive à l'émergence d'un « homme nouveau» (Ciavolella : 127), le Mauritanien, dont Nouakchott représente désormais le présent et l'avenir.

\section{Marginalisation}

5 Alors que la Mauritanie tente de se construire autour de sa capitale, les Fulaabe sont rattrapés par l'extérieur et s'enfoncent dans la marginalité subie. Déjà le pouvoir colonial s'était imposé dans le Karakoro en installant, dès les années 1940, de nouvelles notabilités pour gérer la fiscalité, faisant passer les Fulaabe sous le contrôle des Maures Oulad Zbeyrât. Le Parti unique, créé à l'indépendance et censé se situer au dessus des tribus, s'il incarne la modernité nationale, accentue pour les Fulaabe le processus croissant de marginalisation: seule l'élite y est intégrée, le peuple est oublié. Les Maures nobles de la région deviennent commerçants ou fonctionnaires, ils imposent l'État conquérant à des nomades isolés. La sécheresse de 1970 et surtout de 1983 ruine la frontière fulaabe: c'est la fin de la brousse, des pâturages libres, les terres sont occupées, notamment par les anciens esclaves des Maures (Harâtîn), désormais fixés. La ville où les nomades de tout le pays fuient, l'école, la sédentarisation deviennent les 
références: à une logique d'exclusion se joint alors une logique de dépendance à laquelle les Fulaabe avaient toujours tenté d'échapper.

Nouakchott rassemble tous les déshérités de la brousse. De capitale-repoussoir que personne ne voulait habiter dans les années 1960, on passe à une capitale-refuge dans les années qui suivent. Si jadis les Fulaabe savaient gérer les sécheresses par la mobilité en brousse, aujourd'hui ils créent des quartiers à Kankossa, Mbout ou Kiffa, puis surtout émigrent à Nouakchott où l'ancien ksar fait office de lieu originel d'installation. La capitale est d'abord une ville d'immigrants, de pauvres, les gazra ou occupations illégales se multipliant, quand ce ne sont pas des kebbe ou bidonvilles qui recueillent les nouveaux arrivants. Avec la montée du pouvoir maure dans les années 1970, se caractérisant par la volonté d'ancrage du pays dans le monde arabe et l'arabisation de la société (à l'école ou dans les médias), la communautarisation progresse, engendrant un lent processus de ségrégation entre quartiers et l'apparition de conflits latents entre groupes de population qui rencontreront leur point d'orgue en 1989. Cette ville que les Mauritaniens ont voulue "trait d'union » à l'indépendance devient ainsi la «capitale des Maures » et traduit, tout autant qu'elle exacerbe, les clivages entre Maures et Négro-Mauritaniens.

\section{Le « désordre » de $1989^{3}$}

7 Pour les Fulaabe, "[...] le village ne constitue plus le lieu du bien-être économique... L'espace villageois n'est pas investi de la modernité que l'État espérait incarner ou transposer en brousse » (Ciavolella: 175). Et pourtant c'est là que la marginalité va s'exprimer le plus, les Fulaabe, peu alphabétisés, n'ayant aucun relais dans les réseaux de pouvoir pour atténuer les ferments de l'inégalité qui croît au quotidien. Durant le $20^{e}$ siècle, la vieille idéologie coloniale, alimentant le principe d'une séparation des populations blanches et noires par le fleuve Sénégal, a en partie surdéterminé la création de la Mauritanie indépendante, le déplacement de la frontière entre Sénégal, Mali et Mauritanie (illustrée dans la carte p. 83) puis la remontée des Oulad Zbeyrât, de Kayes à Kankossa, confirmant cet état de fait et encourageant les partisans d'un tel découpage. En février 1989, l'élection d'un maire fulaabe dans une commune de la vallée du Karakoro semble cependant officialiser la prééminence des Peuls sur la zone. Mais, quelques mois plus tard, la violence surgit de l'extérieur, inattendue, et réduit à néant tous les rapprochements antérieurs. Dans le Karakoro (comme dans la haute vallée du fleuve), des vols de bétail sont commis, des villages sont détruits, des gens sont chassés, d'autres sont tués, des papiers d'identité sont déchirés ou brûlés. Les exactions de la part de voisins immédiats ${ }^{4}$ sont rendues possibles par des chefs maures locaux qui excitent et manipulent les Harâtîn, sinon ce sont des étrangers à la zone qui débarquent et s'en prennent aux Fulaabe. Décrites avec précaution et sans voyeurisme aucun, ces violences sont ramenées à leur explication fondamentale : le "plan politique pour l'expropriation de certains groupes » (208), solution aux problèmes du pays ${ }^{5}$, stratégie politique qui trouve son origine dans le centre de la politique mauritanienne et permet de reconstituer la frontière géo-ethnique par la reconduite, encadrée par l'armée, des Fulaabe dans leur supposé pays d'origine, le Sénégal : «peu importe la carte d'identité ou le passeport que vous détenez, c'est votre origine qui est déterminante ", précise à l'époque un fonctionnaire mauritanien cité par R. Ciavolella (215). Là, dans des pages très émouvantes pour ceux qui connaissent cette zone des "trois frontières $\|^{6}$, la marginalisation d'un peuple est extrême et en même temps elle signe l'appartenance 
définitive des Fulaabe à la nation. Les phrases de R. Ciavolella sont justes et soulignent combien l'attention portée aux marges est riche d'enseignements. Il ajoute à propos de ce "désordre» : "c'est le signe que la trajectoire conflictuelle d'un État, qui se réduisait à une élite retranchée dans ses privilèges et à une politique limitée à une diatribe sur l'appartenance, croisait tragiquement la vie de ces populations rurales, brutalement intégrées à une histoire à laquelle elles n'avaient pas participé » (216).

Alors la vie de ces gens change brutalement. En ville, à Nouakchott, la cité se transforme sous les coups de boutoirs de l'inquiétude, du doute, de la suspicion : le ksar se vide de ses populations noires, la médina, les 5 et $6^{\text {es }}$ arrondissements concentrent encore plus les populations originaires de la vallée du fleuve Sénégal, du sud de l'Assaba, du Karakoro (carte d'A. Choplin : 148), ainsi que les immigrés ouest-africains venus à la recherche d'un travail, selon une nouvelle «[...] configuration de la ville relayant les tensions contingentes à l'histoire de la construction de la nation et la marginalisation des populations négro-mauritaniennes» (149) et un processus d'intériorisation de «[...] la distance spatiale qui matérialise une réelle mise à l'écart économique et sociale » (152).

\section{D'une marginalité extérieure à l'exclusion intérieure}

9 Si les mois qui suivent le « désordre » de 1989 voient les Fulaabe réagir, en réactivant le mythe de la mobilité qui sauve par le retour au village, en «[...] votant avec les pieds contre l'État violent» (Ciavolella : 223) par la fuite au Mali, en organisant «[...] des vengeances collectives sans distinction" contre des villages Harâtîn (225), la contestation se fait aussi dans le cadre de l'État devenu légitime ${ }^{7}$. Les Fulaabe se retrouvent intégrés au pays et à la nation tout en étant assignés à des places bien précises, caractéristiques d'une "exclusion intérieure» (Ciavolella: 218). Dans les années 1990, chacun cherche à s'auto-différencier dans la ville, à trouver une place dans l'appareil d'État qui s'ouvre, à se dire arabe pour prendre des postes dans l'administration nationale et locale, critiquant ainsi l'État maure tout en cherchant à l'intégrer, dénonçant les injustices tout en enviant ceux qui profitent des largesses du pouvoir. Entrant pleinement dans le débat politique national, les Fulaabe et leurs représentants acquièrent à ce moment-là une conscience politique tendant à confondre État et ethnie (maure en l'occurrence) et validant par là même l'« obsession étatique d'attribuer une identité à ses sujets... » (221).

L'émigration en ville prend le dessus sur la vie en brousse, certains jeunes Fulaabe intègrent l'université : «l'attraction de la ville devient plus forte que la répulsion de la brousse » précise R. Ciavolella (247), le ladde traditionnel, c'est-à-dire l'inconnu, n'étant plus ce qui est situé au-delà du village mais ce qui est en dehors de Nouakchott ${ }^{8}$. L'islam devient la référence pour une population qui était encore considérée comme païenne au tournant de l'indépendance, il règle les mariages auparavant basés sur la parenté endogamique. En temps d'autochtonie recherchée, afin de ne pas se perdre complètement dans la mondialisation qui ne profite qu'à certains, le lien entre la ville et la brousse fait vivre des réseaux, sans lesquels la capitale, le pays, un mode de vie peut-être, disparaîtraient. La brousse subsiste comme un paradis qui n'est pas encore totalement perdu. "Le village représente la zone de repli et de sécurité pour une population qui a du mal à trouver sa place dans le système mauritanien de la circulation de l'argent facile et du pouvoir monopolisé » (258). Comme si l'inscription 
dans le local, dans le village, permettait une meilleure insertion en ville, dans la communauté, dans les réseaux, dans la vie urbaine, dans les opportunités engendrées par la politique de redistribution par l'élite des quelques miettes de richesse.

11 La cité divisée dont parle A. Choplin (158) est l'illustration d'un pays dans lequel les Fulaabe "[...] partagent avec la plupart de la population mauritanienne, d'origine nomade ou sédentaire, une exclusion de l'accès aux ressources de l'État et d'une économie de plus en plus reliée aux mouvements du capitalisme mondial » (Ciavolella : 261). Là, les deux auteurs se rejoignent quand ils distinguent les «Capitale naabe » ou ressortissants du centre-ville aisé de ceux du $6^{\mathrm{e}}$ arrondissement de Nouakchott, pauvre et populeux, quand ils relèvent la montée de l'économie de la débrouille, du «tcheb tchib ", soulignant par là ce qu'on aperçoit dans nombre de capitale africaines, à savoir l'uniformisation des pratiques de survie chez les jeunes urbains du continent ${ }^{9}$. Dans Nouakchott ô combien pensée et contrôlée par l'État pour être le phare du pays et le lieu où les oppositions sont le plus maîtrisées, ces populations participent à leur façon à la dynamique urbaine. Elles inventent une ville qui n'est pas celle de l'État, ni celle de la Banque mondiale ou des ONG, «[...] les marginaux produisant et étant dotés d'une conscience urbaine et d'une capacité réflexive » (Choplin : 176).

\section{Mauritanie, Nouakchott : un pays et une ville aussi pour les Fulaabe?}

12 Les Fulaabe se disent Fulaabe, parfois Halpulaar ou Négro-Mauritaniens, mais surtout de plus en plus Mauritaniens, vivant au quotidien cette identité-là. Peut-être pourrait-on dire de même à propos de leur rapport à Nouakchott: ils ne se disent jamais « Nouakchottois » mais le deviennent à force d'habiter un des quartiers de la ville, la sociabilité allant «[...] de cour en cour, de thé en thé », selon la belle expression d'A. Choplin (143).

13 Les Fulaabe comme les autres participent à leur manière au "new mauritanian way of life » (Choplin: 133), ostentatoire et à l'opposé de la sobriété de leurs parents. Les jeunes appartiennent au même monde que leurs cousins sénégalais, aux vêtements, à l'allure, au parler, à la musique branchés sur le monde, mais servant, quand il le faut, de repoussoir pour les générations précédentes. Les « ethnoscapes globaux » développés par A. Appadurai ${ }^{10}$ dépassent la brousse, la ville, Kankossa, Kiffa ou Nouakchott, et soustendent l'existence de mondes transnationaux activés par les technologies de la communication dont sont si friands les Mauritaniens. A. Choplin soutient que « [...] les jeunes consomment en ville et consomment la ville» (335), tentent d'avancer à leur place et assument pleinement leur statut de citadins, contribuant à leur façon à donner un visage serein à Nouakchott, capitale «[...] qui se construit lentement dans la multiplicité » (344).

14 À la lecture des deux ouvrages, reste à évaluer les effets à long terme du processus de dépolitisation qui traverse toutes les actions de l'État et qui rend aujourd'hui l'individu, et lui seul, dramatiquement responsable de ses difficultés. A. Choplin rappelle que la pauvreté doit être désormais cachée dans une capitale qui se veut vitrine et où les déshérités, parmi lesquels les Fulaabe, apparaissent comme «inadaptés à la modernité choisie » (265) ou "masse excédante inévitablement exclue du système » (Ciavolella : 378). La « verticalisation » de la ville montre la domination des pouvoirs économique, politique (maure?), sur tous les pauvres de Nouakchott et le peu de marge de 
manœuvre dont ceux-ci disposent dans un monde libéral et globalisé. Arriveront-ils à conquérir des espaces d'autonomie et à construire une idée antagoniste de l'hégémonie, se demande R. Ciavolella à la fin de ce travail ? Pas sûr quand on comprend que les réfugiés du conflit de 1989 au Mali, oubliés de tous et des pouvoirs en place, vivent aujourd'hui une marginalité dans la marginalité et sont en train de disparaître des agendas des bailleurs comme des gouvernements locaux. Pas sûr encore quand on mesure que la marginalité contemporaine vient plus d'un manque de réseaux, de relations dans la capitale, de liens avec la migration internationale, et moins de l'appartenance à tel ou tel monde, fulaabe compris.

\section{Entretien avec les auteurs}

15 - Jérôme Lombard (JL). Vous avez écrit ces deux ouvrages quasiment en même temps : vous êtes-vous vous rendus compte des similitudes, des différences, dans vos démarches, dans votre approche, dans votre façon de décrire le pays?

16 - Ricardo Ciavolella (RC). Plus que des similitudes dans nos ouvrages, je parlerai de complémentarité. Les thématiques sont opposées puisque je pars des marges alors qu'Armelle Choplin part du centre. Mais, au final, comme on ne peut pas comprendre le centre sans les marges et les marges sans le centre, ce n'est pas une réelle opposition. La dialectique est la même (centre-marge) mais abordée de deux angles différents. Nous avons été inspirés par un même sens critique, avec une importance primordiale donnée au terrain. Cet esprit a orienté notre étude des espaces et des sociétés, en poussant à les regarder de différents points de vue : des élites jusqu'aux marginaux, ceux-ci étant plus révélateurs que d'autres. Nous nous sommes lus et indirectement influencés. L'un des points communs à nos recherches est qu'elles portaient initialement sur des questions identitaires (une ville entre monde arabe et Afrique subsaharienne; une société prise dans les oppositions entre "Arabes» et « Négro-africains»). Au fil du terrain et des lectures, nous nous sommes éloignés des discours et de la dimension identitaire pour nous tourner davantage vers des analyses sociales et politiques.

17 - Armelle Choplin (AC). Il est vrai que nos travaux convergent alors que nos cheminements ont été inverses. J'ai découvert le pays depuis l'intérieur pour ensuite arriver à Nouakchott. Riccardo Ciavolella est lui arrivé à Nouakchott et n'est parti que tardivement à l'intérieur. Je n'ai jamais séjourné longtemps en "immersion totale » dans une famille comme lui. En revanche, je suis souvent partie « en brousse » et j'ai sillonné la plupart des régions. Il était pour moi important d'avoir une fine connaissance du pays pour pouvoir comprendre, un tant soit peu, la capitale. Et même si je me suis focalisée sur la capitale, le centre par excellence du pays, j'ai toujours cherché à aller voir au-delà des ministères, à me perdre dans les quartiers périphériques. Au final, je parle de la capitale, certes, mais peut-être plus encore de la ville. Autrement dit, la parole est autant donnée, si ce n'est plus, aux marginaux qui inventent la ville qu'aux élites qui dirigent la capitale. Cette volonté d'aller au plus près des Mauritaniens se ressent dans les deux livres.

18 - JL. Les deux démarches du géographe et de l'anthropologue apparaissent complémentaires. Pouvez-vous décrire quelques éléments de cette proximité?

19 - RC. A mes yeux, la démarche du géographe et de l'anthropologue peuvent être utiles l'une à l'autre. Pour comprendre la vie des pasteurs, j'ai beaucoup lu et utilisé les monographies classiques de géographes qui faisaient état du «genre de vie», du 
rapport à l'environnement, au milieu. Il y a une claire proximité dans le terrain entre les deux disciplines même si nous ne l'envisageons pas de la même façon. Pour le géographe, le terrain est perçu comme un moyen de récupérer de l'information alors que, pour l'anthropologue, le terrain fait déjà partie de sa problématique de recherche. J'ai beaucoup appris des géographes à travers leurs analyses par jeux d'échelles. Leur façon de croiser micro et macro fait leur force et est riche d'enseignements.

20 - AC. Nos démarches sont complémentaires, ce que nous avions d'ailleurs cherché à mettre en évidence dans un article que nous avons coécrit pour un numéro d'Autrepart, consacré à «La ville face à ses marges » $\left(n^{\circ} 45,2008\right)$. J'ai moi aussi beaucoup appris en lisant et en côtoyant les anthropologues. Grâce à eux, j'ai compris comment les catégories sociales et ethniques se sont construites au fil des années de façon exogène puis ont été réappropriées par les individus. Les travaux des anthropologues m'ont permis de sortir des analyses binaires opposant noirs et blancs, Arabes et Africains, musulmans et chrétiens, nomades et sédentaires, État et ethnie, souvent mobilisées de façon simpliste pour expliquer les conflits dans la zone. La complexité du monde mise en évidence par l'anthropologie permet de déconstruire bien des poncifs. Reste après à reconstruire...

21 - JL. Vos deux ouvrages montrent-ils un même pays?

22 - AC. Nos livres parlent d'un même pays mais montrent plusieurs régions, plusieurs quartiers de villes. Ils ne font que rendre compte de sa multiplicité, de ses diverses populations. Le lecteur qui s'attend à lire un énième livre sur les nomades et les dunes de Mauritanie risque d'être déçu. Dans les deux cas, nous nous démarquons de cette image construite par les colons puis entretenue par les dirigeants depuis l'indépendance. J'ai particulièrement cherché à montrer que la Mauritanie ne saurait se résumer au " pays des Maures ». Je voulais parler du monde urbain en pleine mutation, de la richesse de la vallée du fleuve Sénégal, des habitants connectés au reste du monde par France 24 et Al Jazeera, des jeunesses qui ne demandent qu'à s'exprimer...

23 - RC. Les thèmes traités sont différents mais aucun des ouvrages n'a l'intention de présenter de façon exhaustive le pays. De nos démarches relativement similaires, il se dégage des dynamiques constantes en Mauritanie, au centre comme aux marges : fortes inégalités sociales, manipulations politiques, gestion clientéliste des rapports socioéconomiques, importance des relations villes-campagnes, contraste entre le pays des élites et le pays réel.

24 - JL. La marginalité des Peuls est localement transformée à l'aune des ententes avec d'autres groupes eux-mêmes marginalisés mais non fulaabe. Restent-ils en définitive marginaux dans ce pays?

25 - RC. La plupart des Fulaabe restent des marginaux dans la mesure où leurs possibilités de profiter des opportunités économiques, politiques et sociales sont restreintes. Cependant, en jouant avec le concept de "marge ", j'ai souhaité insister, non seulement sur leur marginalisation, mais aussi sur leurs "marges de manœuvre» (agency, stratégies de résistance, formes embryonnaires de mobilisation politique).

26 - J.L. Alors que vous montrez certains signes d'optimisme sur la capacité des Fulaabe ou des « résidus » qui peuplent la capitale à entreprendre ou à se dégager des plages d'autonomie, comment voyez l'avenir des marginaux dans ce pays ?

27 - RC. Mon insistance sur ce que j'ai appelé les "marges de liberté " n'annonce pas forcément une future émancipation de ces populations. Mais, comme nous l'avons écrit 
dans notre article commun, c'est dans les lieux de la marginalité que se joue l'avenir politique et social de la Mauritanie.

- AC. On ne peut vraiment pas dire que la Mauritanie a connu en 2011 son «printemps arabe », mais des soubresauts ont néanmoins eu lieu. De jeunes gens ont investi durant plusieurs jours un terrain vague du centre-ville pour réclamer plus de liberté et davantage de changements. Dans une ville-capitale qui demeure peu politisée et qui n'a même pas une place centrale qui ferait office d'espace public, ces manifestations sont le signe d'une véritable effervescence. Celle-ci se ressent dans les bidonvilles comme en brousse et pourrait, si aucun changement ne se produit, converger à l'avenir vers le centre pour se faire entendre.

\section{BIBLIOGRAPHIE}

Choplin A., 2009. Nouakchott. Au carrefour de la Mauritanie et du monde. Paris, Karthala et PRODIG, $366 \mathrm{p}$.

Ciavolella R., 2010. Les Peuls et l'État en Mauritanie. Paris et Milan, Karthala et UNIMIB, 430 p.

\section{NOTES}

1. Concernant le nombre de Peuls Fulaabe vivant en Mauritanie, Ch. Santoir parle en 1990 de quelques centaines d'individus recensés en 1972 dans la région du Gorgol (haute vallée du fleuve Sénégal), une population tout à fait minoritaire dont la plupart ont émigré depuis 1940 vers l'est. Aucun chiffre aujourd'hui ne permet d'indiquer précisément leur nombre actuel, mais on peut l'estimer à quelques milliers. Cf. Santoir Ch., 1990, « Le conflit mauritano-sénégalais : la genèse. Le cas des Peuls de la haute vallée du Sénégal », Cahiers de sciences humaines, 26 (4), p. 553-576.

2. Brosset D., 1991. Sahara. Un homme sans l'occident. Paris, L'Harmattan, (1ère édition parue en 1935 aux Editions du Moghreb).

3. Les évènements de 1989 sont ainsi dénommés par les Fulaabe. En avril de cette année-là, à l'issue d'une altercation sur la rive droite du fleuve Sénégal entre éleveurs et agriculteurs, deux Sénégalais sont tués par les gardes-frontières mauritaniens. Des émeutes anti-maures se multiplient dans les villages sénégalais de la vallée, puis à Dakar. Les autorités mauritaniennes réagissent avec violence et des populations noires sont agressées, spoliées, parfois tuées ; une partie d'entre elles est chassée de l'autre côté de la frontière sénégalaise, d'autres fuient au Mali. Les violences dégénèrent en un véritable conflit politique entre les deux pays, qui fait au total plusieurs centaines de morts. A la suite de ces graves incidents, les Sénégalais de Mauritanie sont renvoyés et, réciproquement, les Mauritaniens présents au Sénégal expulsés. Cf. Santoir (op. cit.).

4. Précédant ce qui surviendra quelques années plus tard dans l'Afrique des Grands Lacs.

5. Notamment la reconversion économique des Harâtîn.

6. Mali, Mauritanie, Sénégal.

7. Il faut dire aussi que la liquidation physique en 1991-92 de plusieurs centaines d'officiers négro-mauritaniens, suspectés d'avoir organisé une tentative de coup d'État, affaiblit au même moment le potentiel de contestation. 
8. L'auteur fait un parallèle intéressant entre l'évolution de la perception du ladde, au fur et à mesure qu'on s'éloigne de la capitale et qu'on traverse des petites villes, des villages puis des hameaux, et l'utilisation de d'énergies pour la cuisine, passant progressivement de la bonbonne de gaz au feu de bois (250).

9. Cf. notamment Fall A. S., 2008. Bricoler pour survivre. Perceptions de la pauvreté dans l'agglomération urbaine de Dakar. Paris, Karthala ; Simone A., 2004. For the City Yet to Come: Urban Change in Four African Cities. Duke University Press.

10. Appadurai A., 1991, «Global ethnoscapes: notes and queries for a transnational anthropology», in Richard Fox (ed.), Recapturing Anthropology: Working in the Present. Santa Fe, School of American Research Press, p. 191-210.

\section{AUTEUR}

\section{JÉRÔME LOMBARD}

Jérôme Lombard est chercheur à l'IRD. jerome.lombard@ird.fr 